\title{
Computer simulation of the nongyrotropic electron beam-plasma interaction \\ ${ }^{1,3}$ Márcio A. E. de Moraes, ${ }^{2}$ Yoshiharu Omura, ${ }^{3}$ Maria V. Alves, ${ }^{1}$ Universidade de Taubaté, Brazil. ${ }^{2}$ Kyoto University, Japan, ${ }^{3}$ INPE, Brazil
}

Copyright 2003, SBGf - Sociedade Brasileira de Geofísica

This paper was prepared for presentation at the $8^{\text {th }}$ International Congress of The Brazilian Geophysical Society held in Rio de Janeiro, Brazil, 14-18 September 2003.

Contents of this paper were reviewed by The Technical Committee of the $8^{\text {th }}$ International Congress of The Brazilian Geophysical Society and does not necessarily represents any position of the SBGf, its officers or members. Electronic reproduction or storage of any part of this paper for commercial purposes without the written consent of The Brazilian Geophysical Society is prohibited.

\begin{abstract}
In this paper we performed a computer simulation of the nongyrotropic electron beam-plasma interaction based on observational data obtained from ISEE 1 and 2. These data indicated the existence of nongyrotropic electrons just upstream of the Earth's bow shock. In the simulation, the electron beam is assumed to have an extreme nongyrotropy. We study the possible electromagnetic emissions and clarify effects of the nongyrotropy on nonlinear evolution of the electron beam instabilities. In the nongyrotropic case, we found that the magnetic field energy became much larger than in the gyrotropic case, indicating a strong electromagnetic wave emission.
\end{abstract}

\section{Introduction}

Distribution functions in magnetoplasmas of the type $F\left(v_{\square}, v_{\perp}\right)$, where velocities occur both parallel $\left(v_{\square}\right)$ and perpendicular $\left(v_{\perp}\right)$ directions to the background magnetic field $\left(\vec{B}_{0}\right)$ are symmetric with respect to the magnetic field and are termed gyrotropic. When this symmetry is broken, the distribution becomes gyrophase dependent or nongyrotropic (Motschmann et al., 1997).

Nongyrotropic magnetoplasmas with a background magnetic field $\vec{B}_{0}=B_{0} \hat{x}$ have at least one particle population whose unperturbed distribution function depends on the gyrophase angle $\phi=\operatorname{arctg}\left(v_{z} / v_{y}\right)$ (Romeiras et al., 1999\}. The effects of nongyrotropy on linear wave dispersion were first studied in the context of fusion plasmas (Sudan, 1965; Eldridge et al., 1970). Several studies followed these pioneering researches. They showed that the introduction of gyrophase organization (bunching) can bring about coupling among the parallel eingenmodes, with the associated free energy enhancing previously existing (gyrotropic) instabilities or, in otherwise stable media, generating wave growth (Romeiras et al., 1999; Brinca et al., 1992; Brinca et al., 1993; Brinca, 2000).

Nongyrotropic particle populations are frequently encountered in space plasmas. Nongyrotropy has been observed in ion populations in the region at and just upstream of the Earth's bow shock (Thomsen et al., 1985), several Earth radii upstream (Gurgiolo et al.,1981) in the ion foreshock and downstream in the magnetosheath (Sckopke et al., 1990\}. Measurements by the ISEE 1 and ISEE 2 indicate the existence of nongyrotropic electrons in these same regions (Anderson et al., 1985). In a recent work, a possible sustained signature of a non gyrotropic electron distribution just upstream of the Earth's bow shock was obtained by the ISTP WIND 3-D Plasma and Energetic Particle Experiment, showing several examples of electron nongyrotropy (Gurgiolo et al., 2000).

In this work we performed particle simulations of electron beam-plasma interaction in a one-dimensional system taken along the magnetic field. We introduced a nongyrotropy in the particle population of an electron beam drifting against the background plasma. We study possible electromagnetic emissions, and clarify effects of the nongyrotropy on nonlinear evolution of electron beam instabilities. In the nongyrotropic case, we found that the magnetic field energy became much larger than in the gyrotropic case, indicating a strong electromagnetic wave emission

\section{Simulation Model}

We use a particle-in-cell code, KEMPO (Kyoto university ElectroMagnetic Particle cOde) developed at Radio Atmospheric Science Center (Matsumoto and Omura, 1993) that allows spatial variations along the $x$-direction. Since we are interested about parallel propagation, the wave vector of the modes is aligned with the $x$-direction, $\vec{k}=k \hat{x}$, with the ambient magnetic field defined by $\vec{B}_{0}=B_{0} \hat{x}$. Figure 1 shows the reference system used in our simulations

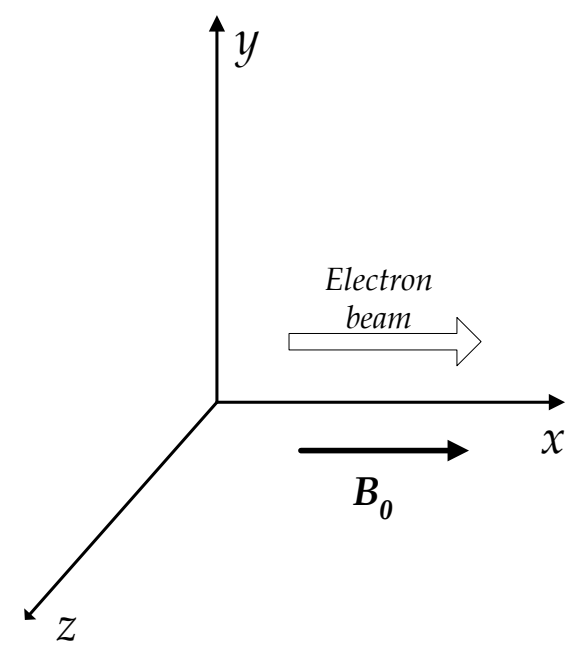

Figure 1 - Reference system used in our simulation. It shows an electron beam propagating parallel to the background magnetic field, both in the $x$-direction of the system. 
For the proposed study the simulation code incorporates three species of charged particles: background electron and ions, and an electron beam with a given drift velocity. We assume the ion species to be of infinite mass, providing a neutralizing background. Both beam and plasma electrons have Maxwelliana population. For gyrotropic (subscript G) and nongyrotropic (subscript NG) cases the electrons of the beam are distributed with a pitch angle $\alpha=60^{\circ}$, where $\alpha=\operatorname{arctg}\left(v_{\perp} / v_{\perp}\right)$ is the angle between background magnetic field and the direction of motion of the particles. For the nongyrotropic case the electron beam velocity component, $v_{y N G}$, is zero $\left(v_{y N G}=0\right)$ and the $v_{z}$ is assumed to have an additional value $v_{z 0} \square 16 v_{\text {the }} \quad v_{z N G}=v_{z G}+v_{z 0}$, introduced at $t=0$. Velocity distribution functions of the moving particles, gyrotropic (top) and nongyrotropic (bottom) cases, are shown in Figure 2, at $t=0$. We can see the formation of a ring for the electron beam, in the gyrotropic case, and an extreme electron beam nongyrotropy with gyrophase angle $\phi=90^{\circ}$, in the nongyrotropic case. Boundary conditions are periodic and preexisting wave packets are not assumed, and all the waves grow self-consistently out of noise.

Electrostatic modes are investigated by observing the longitudinal wave electric fields $(\vec{E} \square \vec{k} \square \hat{x}$ ) whereas the electromagnetic modes by observing the wave field components $\left(E_{y}, E_{z}\right)$, and $\left(B_{y}, B_{z}\right)$.

\section{Results and Discussion}

Simulation results presented in this section were obtained using the parameters shown in Table 1. Parameters were chosen based on observational data from measurements on ISEE 1 and ISEE 2 (Anderson et. al, 1985). The velocities are normalized with respect to $v_{n}$, where $v_{n}=\sqrt{2} v_{t h b}$ (subscript $b$ is related to the nongyrotropic electron beam), and the frequencies are normalized with respect $\left|\Omega_{b}\right|$. The resulting Debye length $\left(\lambda_{D}\right)$ is large enough (in the scale of the grid spacing) to avoid nonphysical heating of the plasma (Birdsal and Langdon, 1985).

Figure 3 presents the time evolution of electrostatic and kinetic energy for the gyrotropic (left) and nongyrotropic (right) case, both in the logarithm scale for the $y$ axis. All the energies were normalized by the initial magnetic energy $\left(B_{0}^{2} / 2 \mu_{0}\right)$. The nongyrotropic case presents higher kinetic and electrostatic energies due to the introduction of $v_{z 0} \neq 0 \square 16 v_{\text {the }}$. We can observe that both cases present similar behavior, the corresponding decreasing of kinetic energy appearing as an increasing of the electrostatic energy, in the beginning of simulation until $t \square 2\left|\Omega_{b}\right|^{-1}$. After this time the kinetic energy became constant, in both cases, and the electrostatic energy decreases slowly.

Table 1 - Values of parameters used in the simulation.

\begin{tabular}{ll}
\hline Parameters & Values \\
\hline Electron plasma frequency $\left(\omega_{p e}\right)$ & $45.4\left|\Omega_{b}\right|$ \\
Electron cyclotron frequency $\left(\Omega_{e}\right)$ & $1.0\left|\Omega_{b}\right|$ \\
electron thermal speed $\left(v_{t h e}\right)$ & $0.0058 c$ \\
electron beam thermal speed $\left(v_{t h b}\right)$ & $0.0146 c$ \\
electron beam drift velocity $\left(V_{d b}\right)$ & $0.1 c$ \\
grid spacing ( $\Delta x)$ & $\approx 2.5 \lambda_{D}$ \\
number of grid points & 4096 \\
number of superparticles & 1638400 \\
time step & $0.00032\left|\Omega_{b}\right|^{-1}$ \\
beam to plasma density ratio $\left(n_{b} / n_{0}\right)$ & 0.05 \\
\hline
\end{tabular}
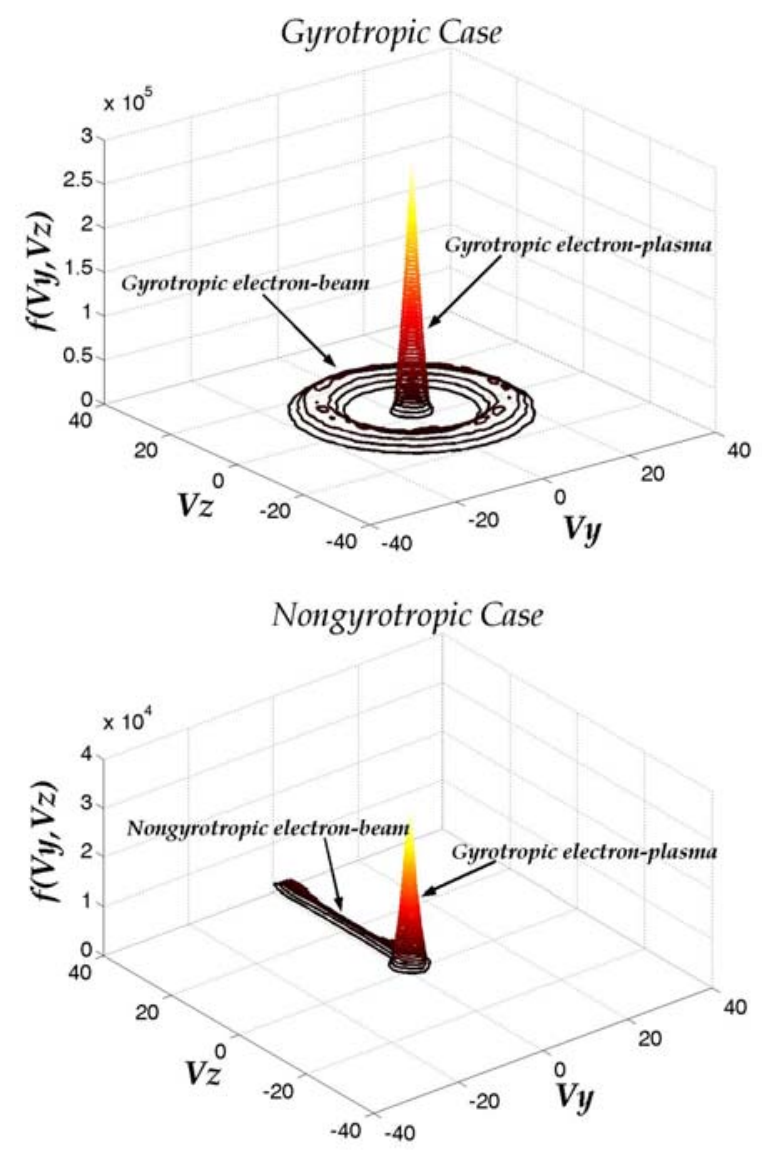

Figure 2 - Velocity distribution functions, at $t=0$, for the gyrotropic (top) and nongyrotropic (bottom) cases.

Concerning the electromagnetic energy, we see that for the gyrotropic case there is no variation along the time, as 
shown in Figure 4 (left), just appearing fluctuations. For the nongyrotropic case, we see an increasing of the electromagnetic energy as shown in Figure 4 (right). The growing of electromagnetic energy starts at $t \square 4\left|\Omega_{b}\right|^{-1}$ reaching the first maximum at $t \square 10\left|\Omega_{b}\right|^{-1}$ (the total time run simulation). This energy gain comes from the electrostatic energy that decreases along the simulation (see the Figure 3).

The diagram $(\omega \times k)$ tells us the modes that are present in the system. We constructed the $(\omega \times k)$ diagram for the electromagnetic fields components $\left(E_{x}, E_{y}, E_{z}, B_{y}, B_{z}\right)$. We will show the $(\omega \times k)$ diagram for the $E_{x}$, and $E_{z}$ components. Figure 5 shows the $(\omega \times k)$ diagram for $E_{x}$ component (electrostatic mode) for the gyrotropic (left) and the nongyrotropic (right) cases, respectively. Colors are related to the intensity of the field component (in $\mathrm{dB}$ ). For both cases we observe Langmuir waves, frequency close to $\square 45\left|\Omega_{b}\right|$, forward and backward propagating and also the beam mode forward propagating in the nongyrotropic case.

Figure 6 shows the $(\omega \times k)$ diagram for the $E_{y}$ component (electromagnetic mode) for the gyrotropic (left) and the nongyrotropic (right) cases. For both cases we observe the RCP high frequency mode, forward and backward propagating. We also observe the whistler mode (RCP low frequency) in both cases. For the nongyrotropic case (right) the whistler mode emission is intensified. Colors are related to the intensity of the field component (in $\mathrm{dB}$ ). We also observe in the nongyrotropic case the presence of an electrostatic mode due the extreme electron nongyrotropy, which behaves like a beam mode.

Figure 7 shows the distribution function of velocity, for the components, $v_{y}$ and $v_{y}$ for different times. This figure illustrates a rotating nongyrotropy with frequency $\Omega_{b}$.

\section{Conclusions}

In this work we performed particle simulations of electron beam-plasma interaction in a one-dimensional system taken along the magnetic field. We introduced a nongyrotropy in the particle population of an electron beam drifting against the background plasma. We compare the behavior of two systems, gyrotropic and nongyrotropic. We observe that at early times, up to $t \square 2\left|\Omega_{b}\right|^{-1}$, both systems have similar behavior. For times larger than $t \square 4\left|\Omega_{b}\right|^{-1}$, there is an enhancement of the electromagnetic energy for the nongyrotropic case. An intensification of the emission of the whistler mode can be observed in the $\omega \times k$ diagram for the $E_{z}$ component (see Figure 6). Different gyrophase angles and density beam to plasma ratios should be investigated in the near future

\section{Acknowledgments}

This work was supported by FAPESP- Fundação Amparo à Pesquisa do Estado de São Paulo, and UNITAU Universidade de Taubaté, Brasil.

\section{References}

A. L. Brinca, J. Atmospheric and Solar-Terrestrial Physics, 62, 701, 2000.

A. L. Brinca, L. Borda de de Água, and D. Winske, Geophys. Res. Let. 12(24), 2445, 1992.

A. L. Brinca, L. Borda de Água, and D. Winske, J. Geophys. Research, 98, 7549, 1993.

C. Gurgiolo, G. K. Parks, B. H. Mauk, C. S. Lin, A. Anderson, R. P. Lin, and H. R, J. Geophys. Research, 86, 4415, 1981.

C. k. Birdsall, and B. Langdon, Plasma Physics via Computer Simulation, McGraw-Hill, NY, 1985.

C. Gurgiolo, D. Larson, R. P. Lin and H. K. Wong, Geophysical Res. Lett., 27, 19, 3153-3156, 2000.

F. J. Romeiras, and A. L. Brinca, J. Geophys. Res.104, 12407, 1999.

K. A. Anderson, R. P. Lin, C. Gurgiolo, G. K. Parks, D. W. Potter, S. Werden, and H. RIUNICODE\{0xe8\}me, J. Geophys. Research, 90, 10809, 1985.

M. F. Thomsen, J. T. Gosling, S. J. Bame, and C. T. Russel, J. Geophys. Research, 90, 267, 1985.

Motschamann, U., Kafemann, H. and Scholer, M. A. Geophysicae, 15, 603, 1997

N. Sckopke, G. Paschmann, A. L. Brinca, C. W. Carlson, and H. LIUNICODE $\{0 x f c\} h r$, J. Geophys. Research, 95, 6337, 1990.

O. Eldridge, Phys. of Plasmas 13, 1791, 1970.

R. N. Sudan, Phys. of Plasmas, 8, 1915, 1965.

Y. Omura, and H. Matsumoto, in: Computer Space Plasma Physics, ed. by $\mathrm{H}$. Matsumoto and $\mathrm{Y}$. Omura, Chap.2, 21-84, 1993. 

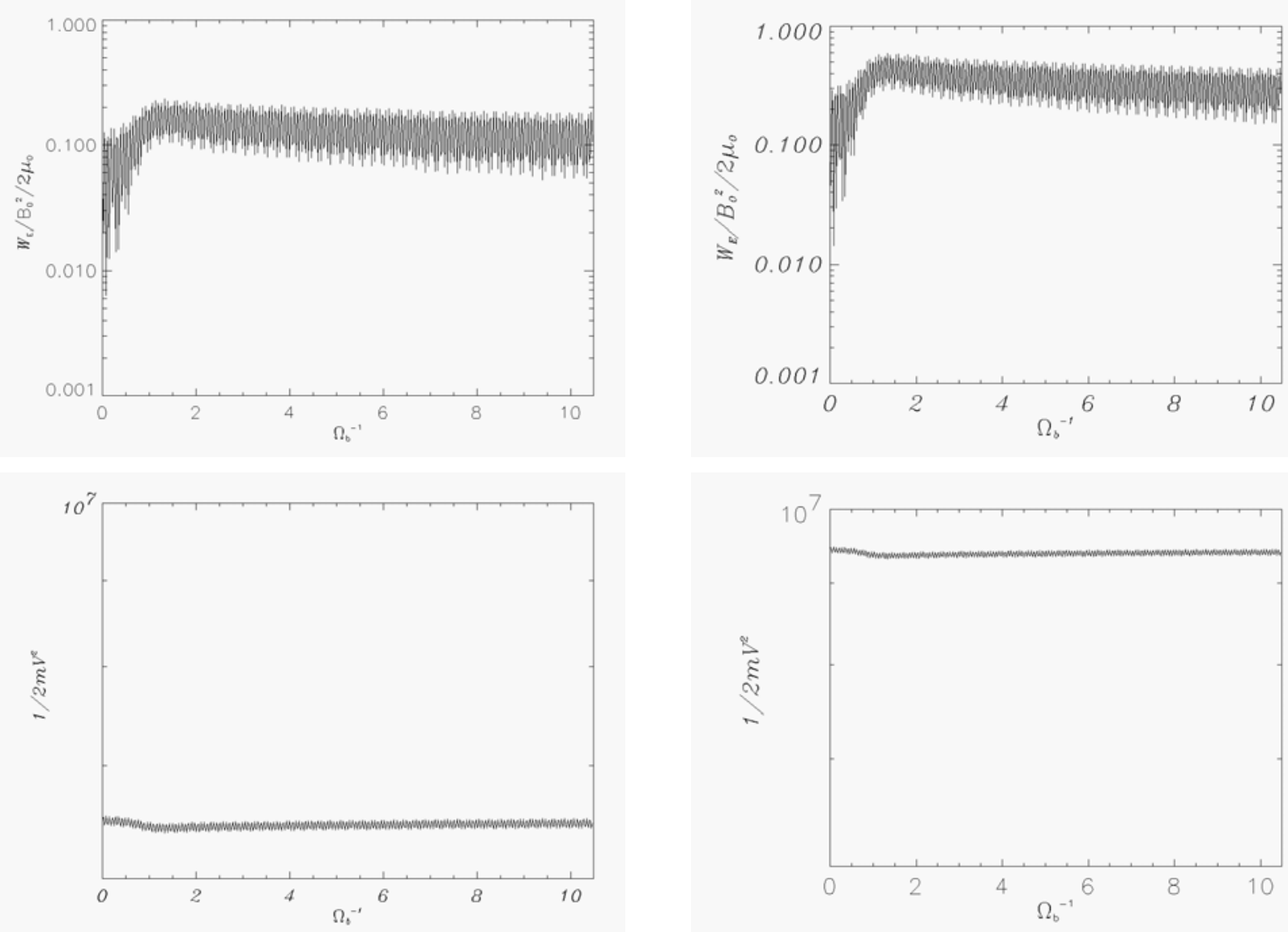

Figure 3 - Time evolution of electrostatic and kinetic energy for the gyrotropic (left) and nongyrotropic (right) cases.
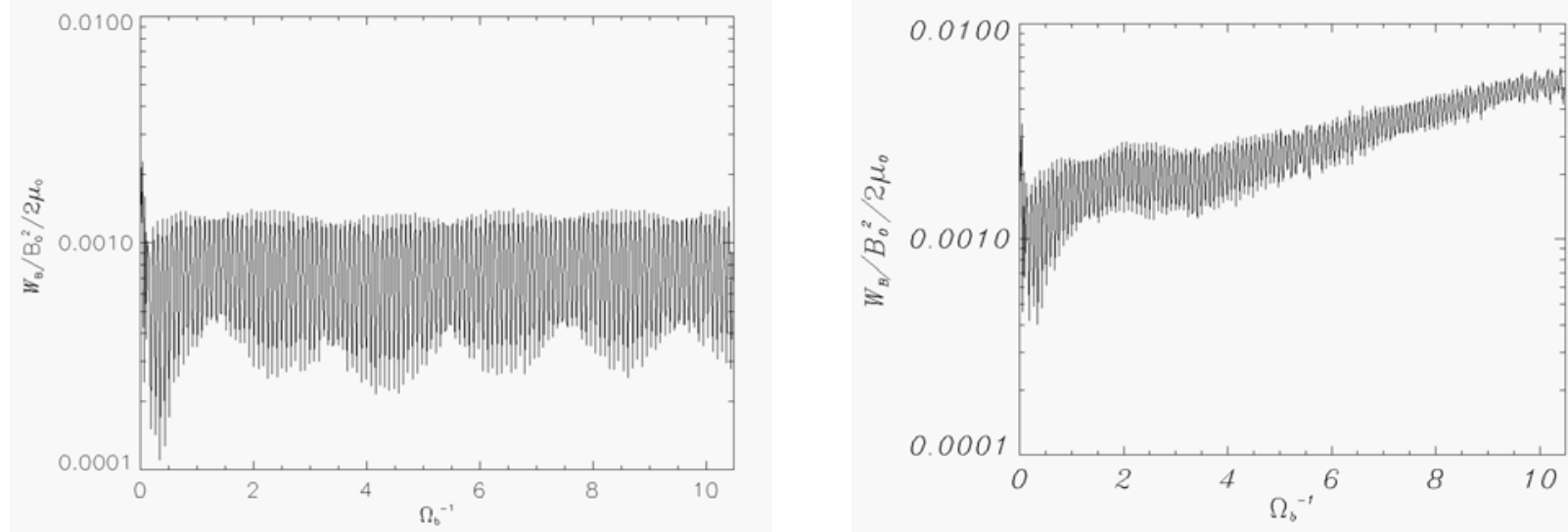

Figure 4 - Time evolution of electromagnetic energy for the gyrotropic (left) and nongyrotropic (right) cases. 

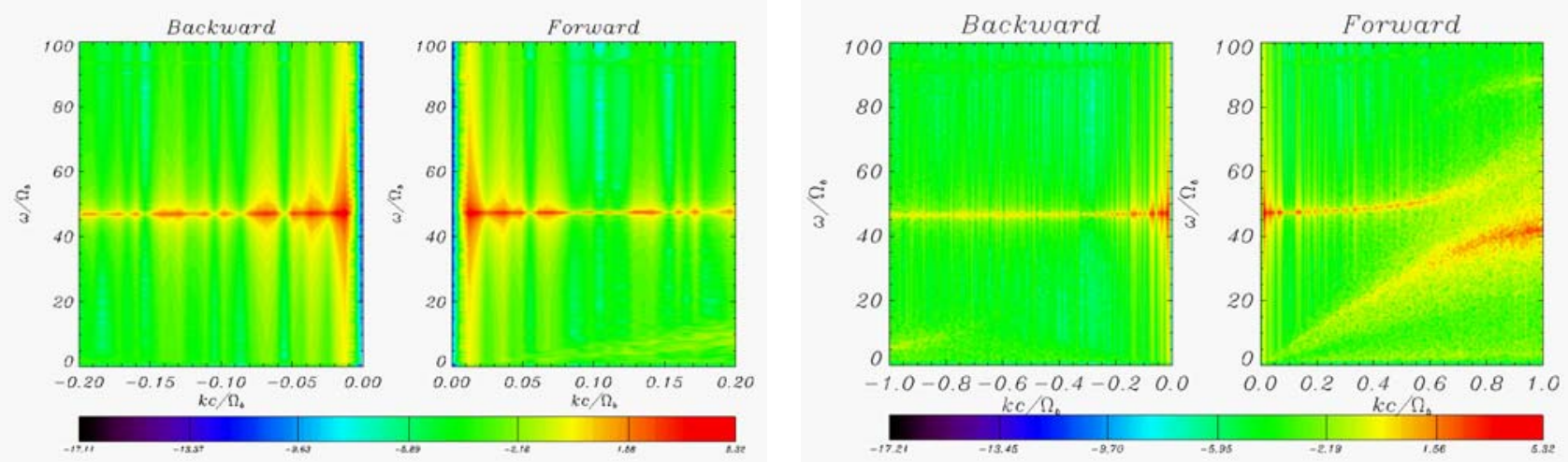

Figure $5-\omega \times k$ diagram for the electric field component, $E_{x}$, electrostatic, for the gyrotropic (left) and the nongyrotropic (right) cases. Colors are related to the amplitude of the component.
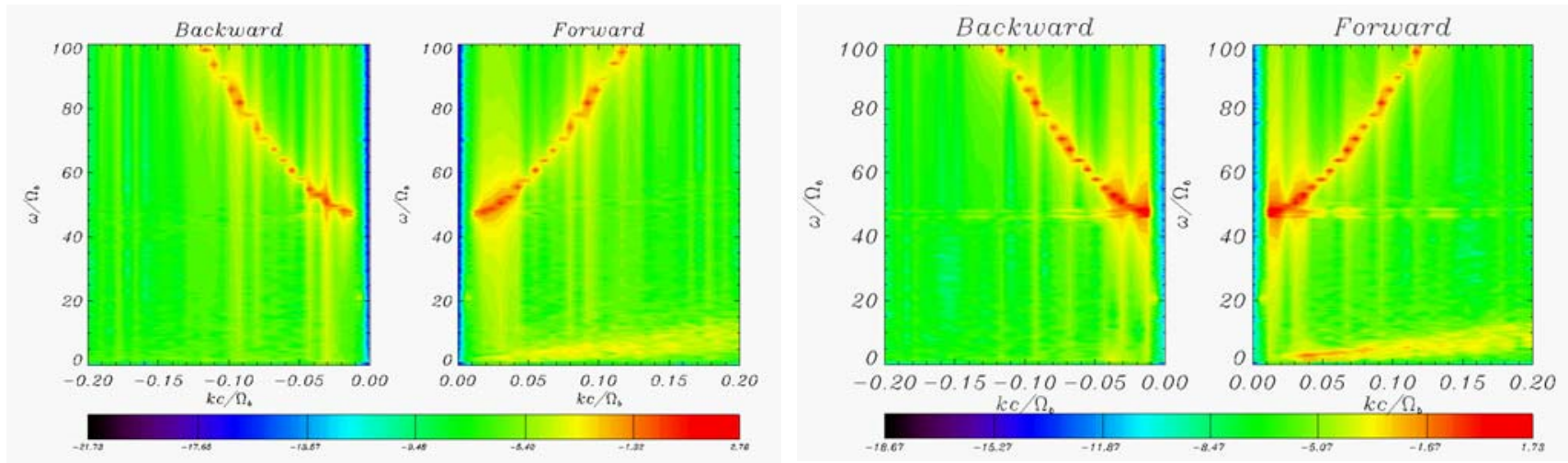

Figure $6-\omega \times k$ diagram for the electric field component, $E_{y}$, electrostatic, for the gyrotropic (left) and the nongyrotropic (right) cases. Colors are related to the amplitude of the component. 

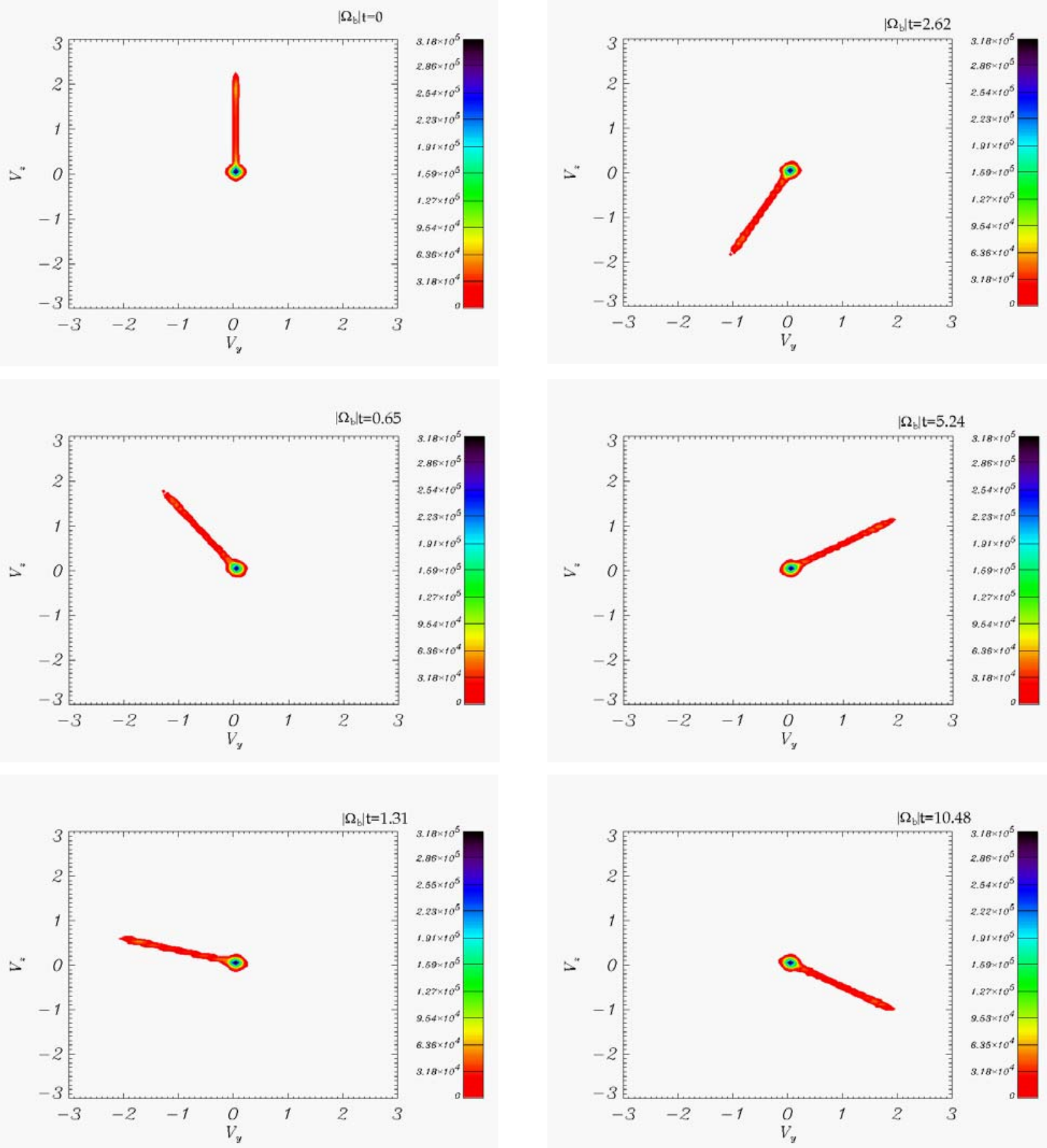

Figure 7 - Contour plots of the distribution function, velocity components $v_{y}$ and $v_{z}$, for the beam and plasma electrons for different time steps, in the nongyrotropic case. 